Acta Crystallographica Section E

Structure Reports

Online

ISSN 1600-5368

David J. Watkin, ${ }^{a} *$ Stephen W. Johnson, ${ }^{b}$ John $H$. Jones ${ }^{b}$ and George W. J. Fleet ${ }^{\text {b }}$

${ }^{a}$ Department of Chemical Crystallography, Chemical Research Laboratory, Mansfield Road, Oxford OX1 3TA, England, and ${ }^{\mathbf{b}}$ Department of Organic Chemistry, Chemical Research Laboratory, Mansfield Road, Oxford OX1 3TA, England

Correspondence e-mail:

david.watkin@chem.ox.ac.uk

\section{Key indicators}

Single-crystal X-ray study

$T=185 \mathrm{~K}$

Mean $\sigma(\mathrm{C}-\mathrm{C})=0.003 \AA$

$R$ factor $=0.051$

$w R$ factor $=0.095$

Data-to-parameter ratio $=10.8$

For details of how these key indicators were automatically derived from the article, see http://journals.iucr.org/e.

\title{
Methyl 2,4-anhydro-5-azido-5,6-dideoxy-L-altronate
}

The title compound, $\mathrm{C}_{7} \mathrm{H}_{11} \mathrm{~N}_{3} \mathrm{O}_{4}$, was prepared from Lrhamnose as a conformationally restricted dipeptide isostere containing an oxetane ring. Its crystal structure was determined to confirm the synthetic product.

\section{Comment}

Sugar amino acids (SAA) are an important class of peptidomimetics (Schweizer, 2002; Gruner et al., 2002). In particular, D-amino acid scaffolds derived from pyranoses (Kriek et al., 2003; El Oualid et al., 2002) and furanoses (van Well et al., 2003; Chakraborty et al., 2002) provide a well established series of conformationally fixed dipeptide isosteres. The azido ester described here, (I), prepared from L-rhamnose, is among the first examples of building blocks for dipeptide isosteres which contain an oxetane ring; it may be viewed as a conformationally restricted dipeptide isostere of L-ala-D-ser, (II).

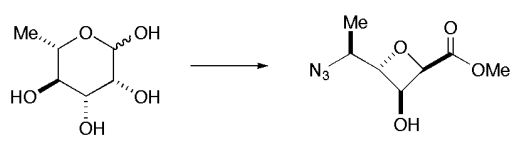

L-rhamnose
(I)

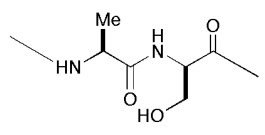

(II)
Fig. 1 shows the asymmetric unit (I). Its absolute structure (C4 $R$ conformation, and C6 and C9 $S$ conformation) was assumed based on the known absolute structure of the starting material.

The crystal packing for (I) consists of slightly pleated ribbons of molecules linked by weak hydrogen bonds, with the sheets stacked in van der Waals contact (Fig. 2).

\section{Experimental}

Compound (I) (Johnson et al., 2004) was recrystallized from chloroform by solvent diffusion with hexane to give colourless plate-shaped crystals.

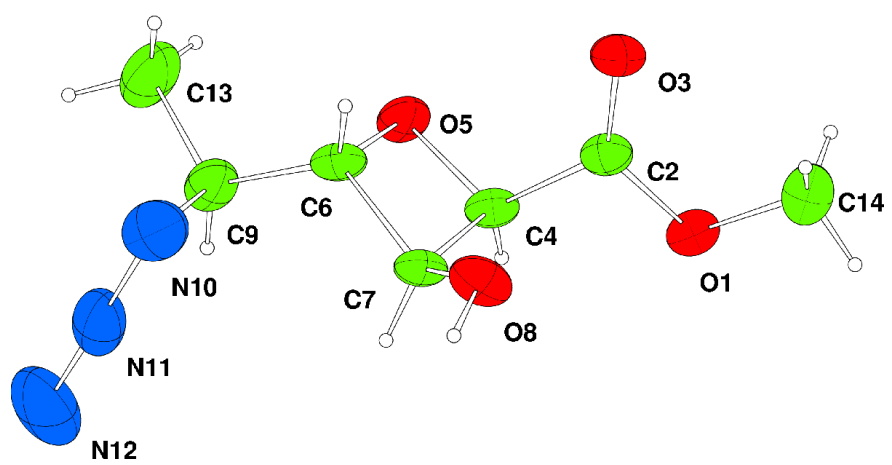

Figure 1

The asymmetric unit of (I), with displacement ellipsoids drawn at the $50 \%$ probability level. H-atom radii are arbitrary.
Received 5 August 2004 Accepted 11 August 2004 Online 28 August 2004
(C) 2004 International Union of Crystallography Printed in Great Britain - all rights reserved 


\author{
Crystal data \\ $\mathrm{C}_{7} \mathrm{H}_{11} \mathrm{~N}_{3} \mathrm{O}_{4}$ \\ $M_{r}=201.18$ \\ Monoclinic, $P 2_{1}$ \\ $a=4.6318$ (2) $\mathrm{A}$ \\ $b=9.8575(5) \AA$ \\ $c=10.6310(6) \AA$ \\ $\beta=92.084(2)^{\circ}$ \\ $V=485.07(4) \AA^{3}$ \\ $Z=2$
}

\section{Data collection}

Nonius KappaCCD diffractometer $\omega$ scans

Absorption correction: multi-scan

DENZO/SCALEPACK (Otwinowski \& Minor, 1997)

$T_{\min }=0.96, T_{\max }=0.98$

4689 measured reflections

\section{Refinement}

Refinement on $F^{2}$

$R\left[F^{2}>2 \sigma\left(F^{2}\right)\right]=0.051$

$w R\left(F^{2}\right)=0.095$

$S=1.01$

1733 reflections

160 parameters

Only coordinates of $\mathrm{H}$ atoms

refined

Table 1

Selected geometric parameters $\left(\AA,^{\circ}\right)$.

\begin{tabular}{lrlr}
\hline $\mathrm{O} 1-\mathrm{C} 2$ & $1.331(2)$ & $\mathrm{C} 6-\mathrm{C} 7$ & $1.533(2)$ \\
$\mathrm{O} 1-\mathrm{C} 14$ & $1.445(3)$ & $\mathrm{C} 6-\mathrm{C} 9$ & $1.521(3)$ \\
$\mathrm{C} 2-\mathrm{O} 3$ & $1.204(2)$ & $\mathrm{C} 7-\mathrm{O} 8$ & $1.405(2)$ \\
$\mathrm{C} 2-\mathrm{C} 4$ & $1.513(3)$ & $\mathrm{C} 9-\mathrm{N} 10$ & $1.486(3)$ \\
$\mathrm{C} 4-\mathrm{O} 5$ & $1.439(2)$ & $\mathrm{C} 9-\mathrm{C} 13$ & $1.515(3)$ \\
$\mathrm{C} 4-\mathrm{C} 7$ & $1.540(2)$ & $\mathrm{N} 10-\mathrm{N} 11$ & $1.234(3)$ \\
$\mathrm{O} 5-\mathrm{C} 6$ & $1.451(2)$ & $\mathrm{N} 11-\mathrm{N} 12$ & $1.132(4)$ \\
& & & \\
$\mathrm{C} 2-\mathrm{O} 1-\mathrm{C} 14$ & $116.48(17)$ & $\mathrm{C} 7-\mathrm{C} 6-\mathrm{C} 9$ & $117.75(15)$ \\
$\mathrm{O} 1-\mathrm{C} 2-\mathrm{O} 3$ & $124.83(18)$ & $\mathrm{C} 4-\mathrm{C} 7-\mathrm{C} 6$ & $84.73(13)$ \\
$\mathrm{O} 1-\mathrm{C} 2-\mathrm{C} 4$ & $110.25(15)$ & $\mathrm{C} 4-\mathrm{C} 7-\mathrm{O} 8$ & $114.53(15)$ \\
$\mathrm{O} 3-\mathrm{C} 2-\mathrm{C} 4$ & $124.92(17)$ & $\mathrm{C} 6-\mathrm{C} 7-\mathrm{O} 8$ & $117.18(15)$ \\
$\mathrm{C} 2-\mathrm{C} 4-\mathrm{O} 5$ & $111.04(14)$ & $\mathrm{C} 6-\mathrm{C} 9-\mathrm{N} 10$ & $105.37(17)$ \\
$\mathrm{C} 2-\mathrm{C} 4-\mathrm{C} 7$ & $114.58(14)$ & $\mathrm{C} 6-\mathrm{C} 9-\mathrm{C} 13$ & $111.88(19)$ \\
$\mathrm{O} 5-\mathrm{C} 4-\mathrm{C} 7$ & $91.58(13)$ & $\mathrm{N} 10-\mathrm{C} 9-\mathrm{C} 13$ & $110.54(19)$ \\
$\mathrm{C} 4-\mathrm{O} 5-\mathrm{C} 6$ & $91.52(12)$ & $\mathrm{C} 9-\mathrm{N} 10-\mathrm{N} 11$ & $113.4(2)$ \\
$\mathrm{O} 5-\mathrm{C} 6-\mathrm{C} 7$ & $91.38(13)$ & $\mathrm{N} 10-\mathrm{N} 11-\mathrm{N} 12$ & $174.7(3)$ \\
$\mathrm{O} 5-\mathrm{C} 6-\mathrm{C} 9$ & $110.23(15)$ & & \\
\hline
\end{tabular}

Table 2

Hydrogen-bonding geometry $\left(\AA{ }^{\circ}\right)$.

\begin{tabular}{lllll}
\hline$D-\mathrm{H} \cdots A$ & $D-\mathrm{H}$ & $\mathrm{H} \cdots A$ & $D \cdots A$ & $D-\mathrm{H} \cdots A$ \\
\hline $\mathrm{O} 8-\mathrm{H} 5 \cdots \mathrm{O}^{\mathrm{i}}$ & $0.82(4)$ & $2.25(3)$ & $2.990(2)$ & $150(3)$ \\
$\mathrm{O} 8-\mathrm{H} 5 \cdots 5^{\mathrm{i}}$ & $0.82(4)$ & $2.32(3)$ & $2.962(2)$ & $135(3)$ \\
\hline
\end{tabular}

Symmetry code: (i) $-x, \frac{1}{2}+y, 1-z$.

Because the intensity data were collected with molybdenum radiation, there were no measurable anomalous differences, as a consequence of which it was admissible to merge Freidel pairs of reflections. The absolute structure of (I) was assumed to correlate with the known absolute structure of the L-rhamnose starting material. All $\mathrm{H}$ atoms were found in difference-density syntheses. They

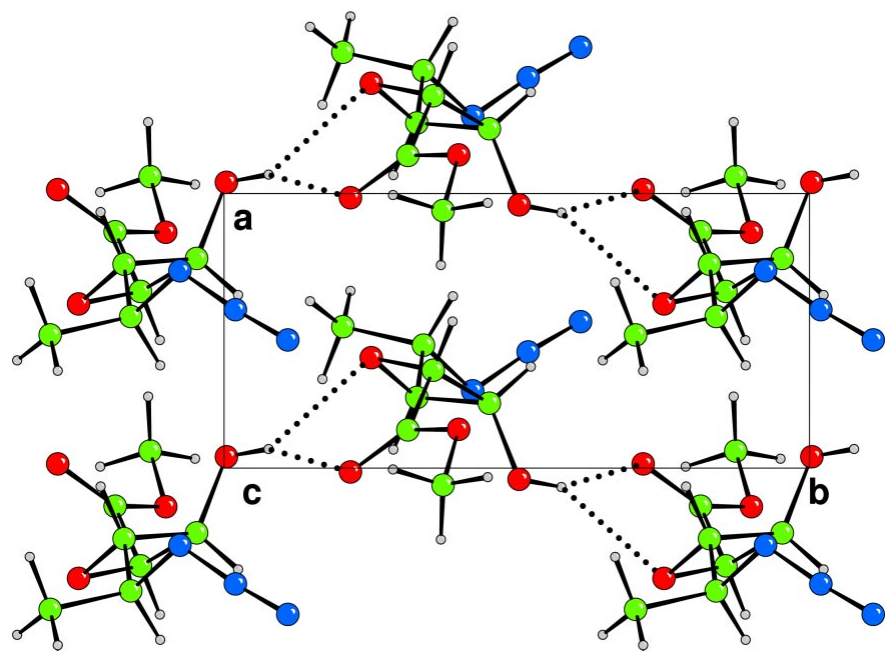

Figure 2

Packing diagram of (I), viewed down the $c$ axis. The weakly hydrogenbonded pleated ribons in the $b c$ plane are simply stacked along the $a$ axis. Hydrogen bonds are shown as dashed lines.

were initially refined with soft restraints on the bonds to regularize their geometry (bond lengths to accepted values, angles either set by symmetry or to accepted values, and $U_{\text {iso }}$ dependent upon the adjacent bonded atom), after which they were refined with riding constraints only.

Data collection: COLLECT (Nonius, 1997-2001); cell refinement: DENZO/SCALEPACK; data reduction: DENZO/SCALEPACK (Otwinowski \& Minor, 1997); program(s) used to solve structure: SIR92 (Altomare et al., 1994); program(s) used to refine structure: CRYSTALS (Betteridge et al., 2003); molecular graphics: CAMERON (Watkin et al., 1996); software used to prepare material for publication: CRYSTALS.

\section{References}

Altomare, A., Cascarano, G., Giacovazzo, C., Guagliardi, A., Burla, M. C., Polidori, G. \& Camalli, M. (1994). J. Appl. Cryst. 27, 435.

Betteridge, P. W., Carruthers, J. R., Cooper, R. I., Prout, K., Watkin, D. J. (2003). J. Appl. Cryst. 36, 1487.

Chakraborty, T. K., Ghosh, S. \& Jayaprakash, S. (2002). Curr. Med. Chem. 9, 421-435.

El Oualid, F., Bruining, L., Leroy, I. M., Cohen, L. H., van Boom, J. H., van der Marel, G. A., Overkleeft, H. S. \& Overhand, M. (2002). Helv. Chim. Acta, 85, 3455-3472.

Gruner, S. A. W., Locardi, E., Lohof, E. \& Kessler, H. (2002). Chem. Rev. 102, 491-514.

Johnson, S. W., Jenkinson, S. F., Angus, D., Jones, J. H., Watkin, D. J. \& Fleet, G. W. J. (2004). Tetrahedron: Asymmetry, 15. In the press.

Kriek, N. M. A. J., van der Hout, E., Kelly, P., van Meijgaarden, K. E., Geluk, A., Ottenhoff, T. H. M., van der Marel, G. A., Overhand, M., van Boom, J. H., Valentijn, A. R. P. M. \& Overkleeft, H. S. (2003). Eur. J. Org. Chem. pp. 2418-2427.

Nonius (1997-2001). COLLECT. Nonius BV, Delft, The Netherlands.

Otwinowski, Z. \& Minor, W. (1997). Methods in Enzymology, Vol. 276, Macromolecular Crystallography, Part A, edited by C. W. Carter Jr and R. M. Sweet, pp. 307-326. New York: Academic Press.

Schweizer, F. (2002) Angew. Chem. Int. Ed. 41, 230-253.

Watkin, D. J., Prout, C. K. \& Pearce, L. J. (1996). CAMERON. Chemical Crystallography Laboratory, Oxford, England.

Well, R. M. van, Marinelli, L., Altona, C., Erkelens, K., Siegal, G., van Raaij, M., Llamas-Saiz, A. L., Kessler, H., Novellino, E., Lavecchia, A., van Boom, J. H. \& Overhand, M. (2003). J. Am. Chem. Soc. 125, 10822-10829. 


\section{supporting information}

Acta Cryst. (2004). E60, o1609-o1610］https://doi.org/10.1107/S1600536804019968]

Methyl 2,4-anhydro-5-azido-5,6-dideoxy-L-altronate

David J. Watkin, Stephen W. Johnson, John H. Jones and George W. J. Fleet

Methyl 2,4-anhydro-5-azido-5,6-dideoxy- $L$-altronate

Crystal data

$\mathrm{C}_{7} \mathrm{H}_{11} \mathrm{~N}_{3} \mathrm{O}_{4}$

$M_{r}=201.18$

Monoclinic, $P 2_{1}$

Hall symbol: P 2yb

$a=4.6318(2) \AA$

$b=9.8575(5) \AA$

$c=10.6310(6) \AA$

$\beta=92.084(2)^{\circ}$

$V=485.07(4) \AA^{3}$

$Z=2$

$F(000)=212$

$D_{\mathrm{x}}=1.377 \mathrm{Mg} \mathrm{m}^{-3}$

Mo $K \alpha$ radiation, $\lambda=0.71073 \AA$

Cell parameters from 1439 reflections

$\theta=5-32^{\circ}$

$\mu=0.11 \mathrm{~mm}^{-1}$

$T=185 \mathrm{~K}$

Plate, colourless

$0.50 \times 0.40 \times 0.20 \mathrm{~mm}$

Data collection

Nonius KappaCCD diffractometer

Graphite monochromator

$\omega$ scans

Absorption correction: multi-scan

DENZO/SCALEPACK (Otwinowski \& Minor, 1997)

$T_{\min }=0.96, T_{\max }=0.98$

4689 measured reflections

1733 independent reflections

1733 reflections with $I>-3 \sigma(I)$

$R_{\text {int }}=0.021$

$\theta_{\max }=32.0^{\circ}, \theta_{\min }=5.3^{\circ}$

$h=-6 \rightarrow 6$

$k=-14 \rightarrow 8$

$l=-15 \rightarrow 15$

Refinement

Refinement on $F^{2}$

Least-squares matrix: full

$R\left[F^{2}>2 \sigma\left(F^{2}\right)\right]=0.051$

$w R\left(F^{2}\right)=0.095$

$S=1.01$

1733 reflections

160 parameters

35 restraints

Primary atom site location: structure-invariant direct methods

Hydrogen site location: difference Fourier map

Only $\mathrm{H}$-atom coordinates refined

$w=1 /\left[\sigma^{2}(F)+(0.034 P)^{2}+0.093 P\right]$, where $P=\left(\max \left(F_{\mathrm{o}}{ }^{2}, 0\right)+2 F_{\mathrm{c}}{ }^{2}\right) / 3$

$(\Delta / \sigma)_{\max }=0.000205$

$\Delta \rho_{\max }=0.22{\mathrm{e} \AA^{-3}}^{-3}$

$\Delta \rho_{\min }=-0.23$ e $\AA^{-3}$

Fractional atomic coordinates and isotropic or equivalent isotropic displacement parameters $\left(\hat{A}^{2}\right)$

\begin{tabular}{lllll}
\hline & $x$ & $y$ & $z$ & $U_{\text {iso }} * / U_{\text {eq }}$ \\
\hline O1 & $0.1410(3)$ & $0.40054(18)$ & $0.21529(13)$ & 0.0400 \\
C2 & $0.1392(4)$ & $0.3140(2)$ & $0.31133(17)$ & 0.0307 \\
O3 & $-0.0147(3)$ & $0.21590(17)$ & $0.31663(13)$ & 0.0401 \\
C4 & $0.3567(4)$ & $0.3571(2)$ & $0.41317(18)$ & 0.0299 \\
O5 & $0.4021(3)$ & $0.25130(15)$ & $0.50505(13)$ & 0.0355
\end{tabular}




$\begin{array}{lllll}\text { C6 } & 0.2570(4) & 0.32935(18) & 0.59975(18) & 0.0320 \\ \text { C7 } & 0.2362(3) & 0.45325(19) & 0.51267(17) & 0.0282 \\ \text { O8 } & -0.0413(3) & 0.50394(17) & 0.48281(16) & 0.0405 \\ \text { C9 } & 0.4480(5) & 0.3407(2) & 0.71873(19) & 0.0401 \\ \text { N10 } & 0.2816(5) & 0.4247(2) & 0.80673(19) & 0.0545 \\ \text { N11 } & 0.4227(5) & 0.5193(3) & 0.85376(19) & 0.0564 \\ \text { N12 } & 0.5334(7) & 0.6089(3) & 0.9016(3) & 0.0819 \\ \text { C13 } & 0.5152(8) & 0.2029(3) & 0.7759(3) & 0.0665 \\ \text { C14 } & -0.0618(5) & 0.3739(3) & 0.1117(2) & 0.0501 \\ \text { H41 } & 0.534(3) & 0.390(2) & 0.3796(16) & 0.0374^{*} \\ \text { H61 } & 0.067(4) & 0.2891(19) & 0.6166(16) & 0.0383^{*} \\ \text { H71 } & 0.370(3) & 0.5239(17) & 0.5357(17) & 0.0350^{*} \\ \text { H91 } & 0.626(4) & 0.391(2) & 0.7011(18) & 0.0519^{*} \\ \text { H131 } & 0.645(5) & 0.216(3) & 0.851(2) & 0.0843^{*} \\ \text { H132 } & 0.324(5) & 0.168(3) & 0.796(2) & 0.0843^{*} \\ \text { H133 } & 0.608(5) & 0.147(3) & 0.711(2) & 0.0843^{*} \\ \text { H141 } & -0.034(5) & 0.450(2) & 0.056(2) & 0.0677^{*} \\ \text { H142 } & -0.261(4) & 0.370(3) & 0.141(2) & 0.0677^{*} \\ \text { H143 } & -0.005(6) & 0.289(2) & 0.076(3) & 0.0677^{*} \\ \text { H5 } & -0.069(6) & 0.576(4) & 0.519(3) & 0.0610^{*}\end{array}$

Atomic displacement parameters $\left(\AA^{2}\right)$

\begin{tabular}{lllllll}
\hline & $U^{11}$ & $U^{22}$ & $U^{33}$ & $U^{12}$ & $U^{13}$ & $U^{23}$ \\
\hline O1 & $0.0484(8)$ & $0.0328(7)$ & $0.0388(7)$ & $-0.0058(6)$ & $0.0007(6)$ & $0.0063(6)$ \\
C2 & $0.0334(8)$ & $0.0237(8)$ & $0.0353(8)$ & $0.0013(7)$ & $0.0067(6)$ & $-0.0004(7)$ \\
O3 & $0.0482(7)$ & $0.0302(7)$ & $0.0419(7)$ & $-0.0118(6)$ & $0.0023(6)$ & $0.0011(6)$ \\
C4 & $0.0287(7)$ & $0.0207(7)$ & $0.0406(9)$ & $0.0018(6)$ & $0.0052(7)$ & $0.0018(6)$ \\
O5 & $0.0472(8)$ & $0.0217(6)$ & $0.0377(7)$ & $0.0108(6)$ & $0.0033(6)$ & $0.0009(5)$ \\
C6 & $0.0369(9)$ & $0.0187(7)$ & $0.0409(9)$ & $0.0007(7)$ & $0.0085(7)$ & $0.0000(7)$ \\
C7 & $0.0260(7)$ & $0.0174(7)$ & $0.0413(9)$ & $0.0006(6)$ & $0.0013(6)$ & $-0.0010(7)$ \\
O8 & $0.0322(7)$ & $0.0285(7)$ & $0.0603(9)$ & $0.0099(5)$ & $-0.0043(6)$ & $-0.0116(7)$ \\
C9 & $0.0530(11)$ & $0.0316(10)$ & $0.0361(9)$ & $0.0099(9)$ & $0.0057(8)$ & $0.0008(8)$ \\
N10 & $0.0712(13)$ & $0.0455(11)$ & $0.0482(11)$ & $0.0071(10)$ & $0.0211(9)$ & $-0.0051(9)$ \\
N11 & $0.0815(15)$ & $0.0483(12)$ & $0.0388(9)$ & $0.0201(11)$ & $-0.0043(9)$ & $-0.0065(9)$ \\
N12 & $0.101(2)$ & $0.0691(18)$ & $0.0741(17)$ & $0.0172(16)$ & $-0.0171(15)$ & $-0.0326(15)$ \\
C13 & $0.106(2)$ & $0.0445(14)$ & $0.0483(13)$ & $0.0222(16)$ & $-0.0035(14)$ & $0.0082(11)$ \\
C14 & $0.0574(13)$ & $0.0535(15)$ & $0.0389(11)$ & $-0.0018(12)$ & $-0.0035(9)$ & $0.0058(10)$ \\
& & & & & &
\end{tabular}

Geometric parameters $\left(\AA,{ }^{o}\right)$

\begin{tabular}{llll}
\hline $\mathrm{O} 1-\mathrm{C} 2$ & $1.331(2)$ & $\mathrm{O} 8-\mathrm{H} 5$ & $0.82(4)$ \\
$\mathrm{O} 1-\mathrm{C} 14$ & $1.445(3)$ & $\mathrm{C} 9-\mathrm{N} 10$ & $1.486(3)$ \\
$\mathrm{C} 2-\mathrm{O} 3$ & $1.204(2)$ & $\mathrm{C} 9-\mathrm{C} 13$ & $1.515(3)$ \\
$\mathrm{C} 2-\mathrm{C} 4$ & $1.513(3)$ & $\mathrm{C} 9-\mathrm{H} 91$ & $0.985(16)$ \\
$\mathrm{C} 4-\mathrm{O} 5$ & $1.439(2)$ & $\mathrm{N} 10-\mathrm{N} 11$ & $1.234(3)$ \\
$\mathrm{C} 4-\mathrm{C} 7$ & $1.540(2)$ & $\mathrm{N} 11-\mathrm{N} 12$ & $1.132(4)$ \\
$\mathrm{C} 4-\mathrm{H} 41$ & $0.964(15)$ & $\mathrm{C} 13-\mathrm{H} 131$ & $0.993(17)$
\end{tabular}




\begin{tabular}{|c|c|c|c|}
\hline $\mathrm{O} 5-\mathrm{C} 6$ & $1.451(2)$ & $\mathrm{C} 13-\mathrm{H} 132$ & $0.981(18)$ \\
\hline $\mathrm{C} 6-\mathrm{C} 7$ & $1.533(2)$ & $\mathrm{C} 13-\mathrm{H} 133$ & $0.996(18)$ \\
\hline $\mathrm{C} 6-\mathrm{C} 9$ & $1.521(3)$ & $\mathrm{C} 14-\mathrm{H} 141$ & $0.969(17)$ \\
\hline C6-H61 & $0.986(15)$ & $\mathrm{C} 14-\mathrm{H} 142$ & $0.983(17)$ \\
\hline $\mathrm{C} 7-\mathrm{O} 8$ & $1.405(2)$ & $\mathrm{C} 14-\mathrm{H} 143$ & $0.962(17)$ \\
\hline $\mathrm{C} 7-\mathrm{H} 71$ & $0.958(16)$ & & \\
\hline $\mathrm{C} 2-\mathrm{O} 1-\mathrm{C} 14$ & $116.48(17)$ & $\mathrm{O} 8-\mathrm{C} 7-\mathrm{H} 71$ & $112.1(10)$ \\
\hline $\mathrm{O} 1-\mathrm{C} 2-\mathrm{O} 3$ & $124.83(18)$ & $\mathrm{C} 7-\mathrm{O} 8-\mathrm{H} 5$ & $111(2)$ \\
\hline $\mathrm{O} 1-\mathrm{C} 2-\mathrm{C} 4$ & $110.25(15)$ & $\mathrm{C} 6-\mathrm{C} 9-\mathrm{N} 10$ & $105.37(17)$ \\
\hline $\mathrm{O} 3-\mathrm{C} 2-\mathrm{C} 4$ & $124.92(17)$ & $\mathrm{C} 6-\mathrm{C} 9-\mathrm{C} 13$ & $111.88(19)$ \\
\hline $\mathrm{C} 2-\mathrm{C} 4-\mathrm{O} 5$ & $111.04(14)$ & $\mathrm{N} 10-\mathrm{C} 9-\mathrm{C} 13$ & $110.54(19)$ \\
\hline $\mathrm{C} 2-\mathrm{C} 4-\mathrm{C} 7$ & $114.58(14)$ & C6-C9-H91 & $110.1(11)$ \\
\hline $\mathrm{O} 5-\mathrm{C} 4-\mathrm{C} 7$ & $91.58(13)$ & N10-C9-H91 & $107.2(12)$ \\
\hline $\mathrm{C} 2-\mathrm{C} 4-\mathrm{H} 41$ & $112.6(10)$ & $\mathrm{C} 13-\mathrm{C} 9-\mathrm{H} 91$ & $111.5(12)$ \\
\hline $\mathrm{O} 5-\mathrm{C} 4-\mathrm{H} 41$ & $113.1(11)$ & C9-N10-N11 & $113.4(2)$ \\
\hline $\mathrm{C} 7-\mathrm{C} 4-\mathrm{H} 41$ & $112.3(11)$ & $\mathrm{N} 10-\mathrm{N} 11-\mathrm{N} 12$ & $174.7(3)$ \\
\hline $\mathrm{C} 4-\mathrm{O} 5-\mathrm{C} 6$ & $91.52(12)$ & C9-C13-H131 & $108.5(19)$ \\
\hline $\mathrm{O} 5-\mathrm{C} 6-\mathrm{C} 7$ & $91.38(13)$ & $\mathrm{C} 9-\mathrm{C} 13-\mathrm{H} 132$ & $103(2)$ \\
\hline $\mathrm{O} 5-\mathrm{C} 6-\mathrm{C} 9$ & $110.23(15)$ & H131-C13-H132 & $113.6(16)$ \\
\hline $\mathrm{C} 7-\mathrm{C} 6-\mathrm{C} 9$ & $117.75(15)$ & $\mathrm{C} 9-\mathrm{C} 13-\mathrm{H} 133$ & $107.9(18)$ \\
\hline $\mathrm{O} 5-\mathrm{C} 6-\mathrm{H} 61$ & $110.5(11)$ & $\mathrm{H} 131-\mathrm{C} 13-\mathrm{H} 133$ & $111.6(16)$ \\
\hline $\mathrm{C} 7-\mathrm{C} 6-\mathrm{H} 61$ & $113.2(11)$ & $\mathrm{H} 132-\mathrm{C} 13-\mathrm{H} 133$ & $111.7(16)$ \\
\hline $\mathrm{C} 9-\mathrm{C} 6-\mathrm{H} 61$ & $111.9(10)$ & $\mathrm{O} 1-\mathrm{C} 14-\mathrm{H} 141$ & $103.0(17)$ \\
\hline $\mathrm{C} 4-\mathrm{C} 7-\mathrm{C} 6$ & $84.73(13)$ & $\mathrm{O} 1-\mathrm{C} 14-\mathrm{H} 142$ & $110.9(16)$ \\
\hline $\mathrm{C} 4-\mathrm{C} 7-\mathrm{O} 8$ & $114.53(15)$ & $\mathrm{H} 141-\mathrm{C} 14-\mathrm{H} 142$ & $111.6(16)$ \\
\hline $\mathrm{C} 6-\mathrm{C} 7-\mathrm{O} 8$ & $117.18(15)$ & $\mathrm{O} 1-\mathrm{C} 14-\mathrm{H} 143$ & $106.2(17)$ \\
\hline $\mathrm{C} 4-\mathrm{C} 7-\mathrm{H} 71$ & $112.1(11)$ & $\mathrm{H} 141-\mathrm{C} 14-\mathrm{H} 143$ & $113.4(15)$ \\
\hline $\mathrm{C} 6-\mathrm{C} 7-\mathrm{H} 71$ & $113.5(11)$ & $\mathrm{H} 142-\mathrm{C} 14-\mathrm{H} 143$ & $111.4(16)$ \\
\hline
\end{tabular}

Hydrogen-bond geometry $\left(\AA,{ }^{\circ}\right)$

\begin{tabular}{lllll}
\hline$D-\mathrm{H} \cdots A$ & $D-\mathrm{H}$ & $\mathrm{H} \cdots A$ & $D \cdots A$ & $D-\mathrm{H} \cdots A$ \\
\hline $\mathrm{O} 8-\mathrm{H} 5 \cdots \mathrm{O} 3^{\mathrm{i}}$ & $0.82(4)$ & $2.25(3)$ & $2.990(2)$ & $150(3)$ \\
$\mathrm{O} 8-\mathrm{H} 5 \cdots \mathrm{O} 5^{\mathrm{i}}$ & $0.82(4)$ & $2.32(3)$ & $2.962(2)$ & $135(3)$ \\
\hline
\end{tabular}

Symmetry code: (i) $-x, y+1 / 2,-z+1$. 